\title{
LFM Radar Convolution Jamming Suppression Based on Oblique Projection in FrFT Domain
}

\author{
Wang Jun, Ping Fulong, Zhao Yuan and Tang Bin \\ School of Electronic Engineering, UEST of China, Sichuan Chengdu 611731
}

\begin{abstract}
Convolution false-targets jamming against LFM fire-control radars generates range and velocity false targets which are coherent with target echo, which increases the difficulty of jamming detection and suppression and makes the victim radar system lost the track of real target. To combat against this type of jamming, the uncorrelated characteristic between the jamming and echo in FRFT domain is discussed firstly. Thus, an oblique projection operator which is capable of suppress convolution false-targets jamming is built. The simulation results showed the operator have little effect on the echo and proved the effectiveness of this method.
\end{abstract}

\section{Introduction}

The function of fire-control radar system is targets tracking through their range and velocity information. This radar system concentrates on one target only when process tracking. Electronic countermeasure (ECM) techniques against fire-control radar systems are enhanced by using digital radio frequency memory (DRFM), which is a challenge for radar systems to combat the impact of DRFM. With the application of DRFM, convolution false targets jamming can be applied as a simple ECM scheme to deceive fire-control radar, therefore making it hard to distinguish the real target from the false one. It also decreases the tracking performance and even makes the system fail to track the real target [1]. Moreover, convolution jamming can also generate multi-targets after CFAR processing, which increases the calculation burden for data processing and blankets the echo from the real target. It is noted that, range-gate pull off (RGPO), if analysed in one PRI, can also be regarded as convolution false range target jamming. RGPO makes fire control radar lost its track from real target and turn to track the false one. Thus, the application of convolution false target jamming against fire-control radars makes the usage of Electronic counter countermeasure (ECCM) with false target suppression performance in the radar systems an exigency.

In order to suppress active false target jamming, Luo [2] proposed the oblique projection based on the uncorrected feature between SMSP jamming and echo signal, by building a projection operator to suppress the jamming. Lu [3] studied pulse diversity to cancel range and velocity false target jamming. This method shows an outstanding property. But it is still a puzzle to cancel jamming when jamming is over-lapped with echo in time or frequency domain.
This paper proposed a jamming suppression method to combat against convolution false target jamming based on the assumption that the radar system has detected the echo through jamming detection. Fortunately, a few researches have focused on LFM active jamming detection like ref. [4]. Based on the previous researches, this paper discusses the uncorrelated characteristic between echoes and jamming based the "thumbtack" feature of LFM signal in FRFT domain [5]. Finally, build an oblique projection operator according to [6] and [7] to suppress the jamming. With the application of the oblique projection, jamming suppression can be processed instantaneously. The paper is organized as follows: Section II models target echo and convolution jamming. Section III proves the uncorrelated property and built an oblique projection operator, the procedure of this method is also shown in this section. Section IV shows the simulation results.

\section{Convolution jamming modelling}

Consider the setup where the fire-control radar transmits LFM signal for moving target detection. The jammer intercepts and re-transmits modulated jamming to combat fire-control radar system. The modulation can be in time delay, frequency shift and even both.

Herein, $S(t)$ represents the real target echo. In the time domain, $S(t)$ is defined as

$$
\begin{aligned}
& S(t)=\operatorname{rec}\left(\frac{t-\tau}{T}\right) \cdot \exp \left(j \phi_{0}\right) . \\
& \exp \left(j 2 \pi f_{c}(t-\tau-\Delta \tau)+j k \pi(t-\tau-\Delta \tau)^{2}\right)
\end{aligned}
$$


where $\varphi_{0}$ is the initial phase, $f_{c}$ is the carrier frequency of transmitted LFM signal, $f_{d}(t)$ denotes the Doppler frequency of real target. $k=B / T_{p}$ denotes the chirp rate, $B$ and $T_{p}$ represents the band-width and pulse-width respectively. $\tau=2 R(t) / c$ denotes the delay of echo, while $R(t)$ is the range function of the real target. To simplify the derivation, suppose $\varphi_{0}=0$, and the Doppler frequency $f_{d}(t)$ is constant for the real target.

The transmitted jamming is

$$
J(t)=S(t) * f(t)
$$

where $f(t)$ represents the modulation function of the convolution jamming and $*$ denotes the convolution operation.

For range-velocity false target

$$
f(t)=\sum_{i=1}^{N_{i}} \delta\left(t-\tau_{i}\right) \exp \left(j 2 \pi f_{d_{i}} t\right)
$$

where $N_{i}$ represents the ith false target, $\tau_{i}$ and $f_{d_{i}}$ represents the range and velocity of $i t h$ false target, respectively. While $\delta(t)$ is the Dirichlet function. Substitute equ.(3) into equ.(2),

$$
J(t)=\sum_{i=1}^{N_{j}} S\left(t-\tau_{i}\right) \exp \left(j 2 \pi f_{d_{i}} t\right)
$$

the received signal of fire-control radar can be represented as,

$$
S_{r}(t)=S(t)+J(t)+n(t)
$$

where $n(t)$ represents the Gaussian white noise , $f(t)$ represents the type of jamming, it can be in range, velocity or the range-velocity convolution jamming.

\section{Oblique projection operator modelling}

\subsection{Basis of Fractional Fourier Transform}

Convolution jamming and echo usually over-lap in time and frequency domain. Detection can only indicate the position of echo. But when jamming and echo overlapped in time domain, there will be two target indications in one range gate, and then it is difficult to suppress the jamming simply. Thus, it is necessary to suppress the jamming through signal processing perspective. However, after rotating the "optimal angle" in a fractional domain, the LFM signal will show a "thumbtack" property. According to [5], when rotating the optimal angle $\alpha$, the definition of $S(t)$ in FrFT domain is given by

$$
\begin{aligned}
& S^{\alpha}(u)=A_{\alpha} \exp \left(j \pi u^{2} \cot \alpha\right) . \\
& T \frac{\sin \left[\pi\left(f_{0}-u \csc \alpha\right) T\right]}{\pi\left(f_{0}-u \csc \alpha\right) T}
\end{aligned}
$$

$$
\begin{aligned}
& A_{\alpha}=\frac{\exp [-j \pi \operatorname{sgn}(\sin \alpha) / 4+j \alpha / 2]}{|\sin \alpha|^{1 / 2}}, \\
& f_{0}=f_{c}+f_{d}
\end{aligned}
$$

Similarly, the corresponding expression of convolution jamming $J(t)$ in FrFT domain can be represented by

$$
\begin{gathered}
J^{\alpha}(u)=A_{\alpha} \exp \left(j \pi u^{2} \cot \alpha\right) T . \\
\sum_{i=1}^{N_{j}} \frac{\sin \left[\pi\left(f_{0}+\Delta f-u \csc \alpha\right) T\right]}{\pi\left(f_{0}+\Delta f-u \csc \alpha\right) T}
\end{gathered}
$$

where $\Delta f=f_{d j}-k \tau_{j} \cdot f_{d j}$ and $\tau_{j}$ denotes the Doppler frequency and delay of false target, respectively.

Note that in equ.(8), the derivations have the same structure which are the range and velocity false target. This united structure proves that in FrFT domain, the range false target acts the same way as the velocity false target.

According to [8], the definition of fractional order cross-correlation is

$$
\begin{aligned}
& \left(S \otimes_{\alpha} J\right)(v)=e^{j 2 \pi\left(v^{2} / 2\right) \cos \alpha \sin \alpha} . \\
& \int S(t) J^{*}(t-v \cos \alpha) e^{j 2 \pi t v \sin \alpha} d t
\end{aligned}
$$

where $\otimes_{\alpha}$ denote fractional convolution in rotate angel $\alpha$

To simplify the derivation, suppose the delay of echo is zero and the carrier frequency is also set to zero, substitute equ.(1) and equ.(2) into equ.(9).

$$
\begin{aligned}
& R_{\alpha}(v)=\left(S \otimes_{\alpha} J\right)(v)=e^{j \pi v^{2}(\cos \alpha \sin \alpha-\cos \alpha)+j \pi k \tau^{2}} . \\
& \int_{0}^{T} \exp \{j \pi[2 v(\cos \alpha-\sin \alpha)-2 k \tau] t\} d t
\end{aligned}
$$

Define

$$
\rho_{\alpha}=\frac{R_{\alpha}(0)}{\sqrt{\left\|S_{\alpha}(v)\right\|_{2}\left\|J_{\alpha}(v)\right\|_{2}}}
$$

as fractional cross correlation coefficient between echo and convolution jamming, $\|\cdot\|_{2}$ represents 2-Norm of a vector. According to [9], a traditional DRFM jammer will bring an extra time delay greater than 40ns. Assume there is only one convolution range false target, which has the minimized delay $\tau$. Substitute this minimized delay into equ.(11) and it is easy to find out that $\rho_{\alpha}$ is approximates to be zero. Namely, echo and convolution jamming is uncorrelated in FRFT domain. It is observed in (8) that velocity, range-velocity false target act the same in FrFT domain, thus, the fractional cross correlation between convolution jamming and echo is approximately to be zero.

\subsection{Oblique Projection Operator}

Behrens and Scharf discussed the suppressions of unrespect signal using subspace oblique projection in 
$[6,7]$.The projection operator is capable of suppressing the undesired component without information loss.

Define $V$ as a Hilbert space. $H$ is the sub-space of $V$ and $H=H_{1} \oplus H_{2}$, where $\oplus$ denotes the direct sum of sub-space. Herein, $H_{1}$ and $H_{2}$ is uncorrelated (disjoint) sub-space of $H . S \in \mathbb{C}^{n \times m}$ and $J \in \mathbb{C}^{n \times k}$ are two matrix with full column rank. The range of $S$ is $H_{1}$ and the range of $J$ is $H_{2}$, they are disjoint.

As defined in $[6,7]$, the oblique projection operator is

$$
\begin{aligned}
& E_{J \mid S}=J\left(J^{H} P_{S}^{\perp} J\right)^{-1} J^{H} P_{S}^{\perp} \\
& E_{S \mid J}=S\left(S^{H} P_{J}^{\perp} S\right)^{-1} S^{H} P_{J}^{\perp}
\end{aligned}
$$

where $P^{\perp}$ is orthogonal projector.

The received signal in FrFT domain can be represented as

$$
S_{r}^{\alpha}(u)=S^{\alpha}(u)+J^{\alpha}(u)+\operatorname{FrFT}(n(t))
$$

Fire-control radar system usually focus on only one target when process target tracking, based on the theory of oblique projection, the desired echo sub-space is the span of column vector

$$
A=\operatorname{span}\left\{S^{\alpha}\right\} \quad S^{\alpha} \in C^{n \times 1}
$$

Similarly, the undesired jamming sub-space is the span of range and velocity false target jamming rotated $\alpha$

$$
B=\operatorname{span}\left\{J^{\alpha}\right\} \quad J^{\alpha} \in C^{n \times 1}
$$

According to Section III, $S^{\alpha}$ is uncorrelated with $J^{\alpha}$. Usually, it is impossible to obtain the orthogonal projector $P^{\perp}$. Thus, according to [10], the statically subspace oblique projection operator is,

$$
E_{A \mid B}=A\left(A^{H} R_{B}^{+} A\right)^{-1} A^{*} R_{B}^{+}
$$

where, $R_{s_{r}}=E\left[S_{r}^{\alpha}(u) \times\left(S_{r}^{\alpha}(u)^{H}\right)\right],(\cdot)^{+}$represents The Moore-Penrose Inverse. The formation of operator requires the information of received signal and target component. The range of the oblique projection operator $E_{A \mid B}$ is $S^{\alpha}(u)$.And the null space of $E_{A \mid B}$ is $J^{\alpha}(u)$. Namely

$$
E_{A B} \cdot S^{\alpha}=S^{\alpha}, E_{A B} \cdot J^{\alpha} \rightarrow 0
$$

According to the orthogonality property between noise space and signal space (including echo and jamming)

$$
E_{A B} \cdot \operatorname{FrFT}(n(t)) \rightarrow 0
$$

$\operatorname{Equ}(17)$ and equ(18) show that the estimation of echo in FrFT domain is

$$
\hat{S}^{\alpha}(u)=E_{A B} \cdot S_{r}^{\alpha}(u) \rightarrow S^{\alpha}(u)
$$

\subsection{Procedure of Convolution Jamming Suppression}

Step1.Fractional Fourier Transform of received signal according to equ.(6) and equ.(8).

Step2. Target detection and echo extraction

Suppose delay and Doppler frequency of real target is well estimated, the echo reaches its peak at $u_{0}$ in the FrFT domain. We can then extract target component in FrFT domain through fractional filtering. The residue is applied to estimate jamming component.

Step3.Formation of oblique projection operator based on equ.(16) and equ.(19).

Step4.Processing inverse FrFT to re-construct echo

Step5.Jamming suppression evaluation

Signal to noise jamming ratio improvement factor $I_{S J N R}$ is applied to evaluate the effect of the oblique projection operator. $I_{S J N R}$ defined as

$$
\begin{aligned}
& I_{\text {sjnr }}=S J N R_{\text {out }}-S J N R_{\text {in }} \\
& =10 \log \left(\frac{\|\mathbf{A} \theta\|_{2}^{2}}{\|y-\mathbf{A} \theta\|_{2}^{2}}\right)-10 \log \left(\frac{\|\mathbf{A} \theta\|_{2}^{2}}{\|x-\mathbf{A} \theta\|_{2}^{2}}\right)
\end{aligned}
$$

The likelihood factor is applied to evaluate the distortion after jamming suppression.

$$
f_{l}=\frac{S_{t}(t) \cdot S_{e}^{*}(t)}{\sqrt{\left|S_{t}(t)\right|^{2} \cdot\left|S_{e}(t)\right|^{2}}}
$$

The simulations are carried out in order to verify the effective of the method proposed.

\section{Simulations}

\subsection{Range False Target Suppression}

The simulations are carried out in the following scenario, the bandwidth of transmitted LFM signal $B=5 \mathrm{MHz}$ and the pulse-width $T_{p}=10 \mu \mathrm{s}$. There exist three false targets $100 \mathrm{~m}, 300 \mathrm{~m}$ and $750 \mathrm{~m}$ away from the real target. It is noted that the target $100 \mathrm{~m}$ away from the real target falls in the same range gate, thus it is impossible to cancel the jamming through range gate. These false targets have the same Doppler frequency as the echo. Fig.1 shows the improvement factor of signal to jamming ratio $I_{S J N R}$ through 100 times Monte Carlo experiment when SJR is $0 d B$ and $-3 d B$, while SNR ranges from $-6 d B$ to $6 d B$ with step of $1 d B$. 


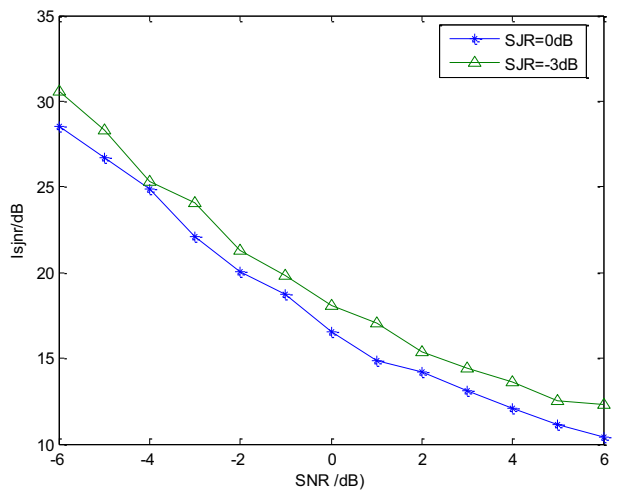

Figure 1. The improvement factor

From the simulation result shown in Fig.1, this method is valid to suppress the Range false targets and improve SJNR. However $I_{S J N R}$ shows a downward trend with SNR which is reasonable because the oblique projection operator can suppress noise simultaneously.

Fig. 2 shows the likelihood of target echo indicates the distortion after jamming suppression with the same simulation scenario

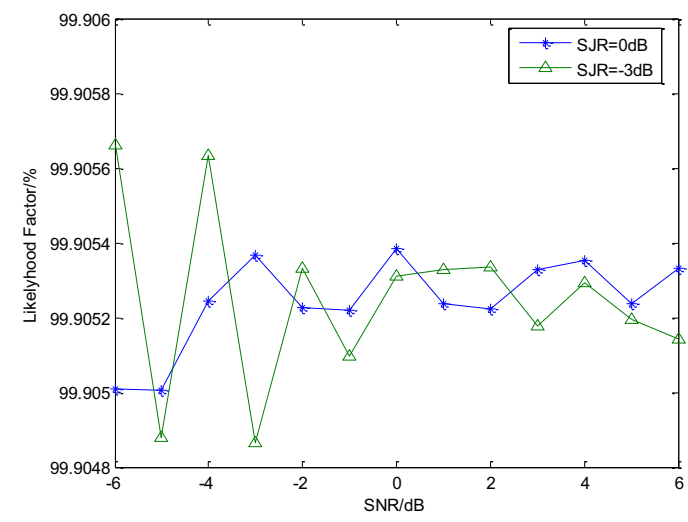

Figure 2. Likelihood factor

Fig. 2 shows the echo after jamming suppression does not have too much distortion. The likelihood factor remains approximately 1 which indicates that the projection operator is capable of preserve the echo component through jamming suppression.

\subsection{Range-Velocity Joint False Targets Suppression}

The simulations are carried out in the following scenario: The bandwidth of Radar and the pulse duration is the same as above, to simplify the analysis, supposing the carrier frequency of the fire-control radar is $1 \mathrm{GHz}$. There exist three range-velocity joint false targets at $300 \mathrm{~m}$, $750 \mathrm{~m}$ and $2400 \mathrm{~m}$ away from echo. However, these false targets have the same Doppler frequency equals to $2 \mathrm{KHz}$.

Fig.3 shows the improvement factor of signal to jamming ratio $I_{S J N R}$ through the 100 times of Monte Carlo experiment under which the SJR is $0 d B$ and $-3 d B$, while SNR ranges from $-6 d B$ to $6 d B$ with a step of $1 d B$.

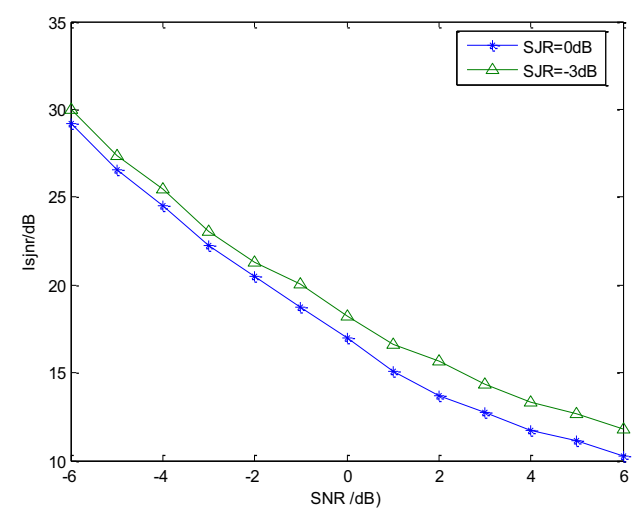

Figure 3. The improvement factor

According to Fig.3, this method is also valid for range-velocity joint jamming suppression. Same as range false target suppression, this improvement factor shows a downward trend.

Simulation in Fig.4 shows the likelihood of target echo.

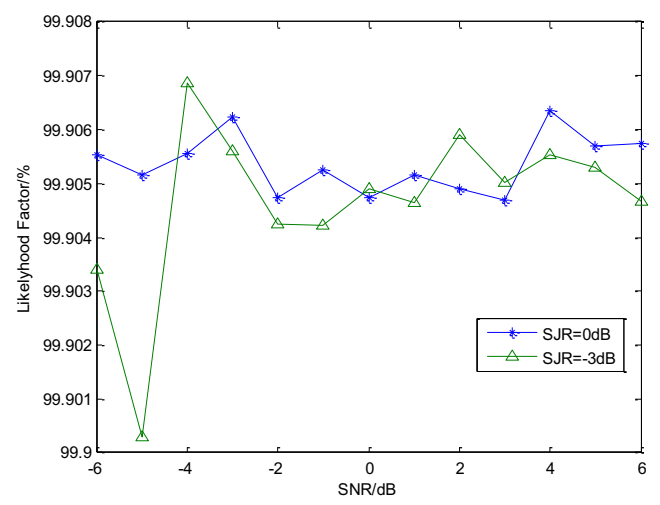

Figure 4. Likelihood factor

Fig.4 shows the signal after jamming suppression which does not have too much distortion.

\section{Conclusion}

In this paper, the technique based on the oblique projection operators in FRFT domain is proposed. Suppression of convolution jamming and noise can be realized simultaneously. Once a target is detected in the range-gate or velocity-gate of the fire-control radar, the information in FRFT domain can be obtained. The simulation results indicate that the method based on oblique projection in FRFT domain is efficient for false target suppression.

\section{Acknowledgment}

This work Project supported by the National Defense Pre-Research Foundation of China (NO.9140A07030713DZ02101). 


\section{References}

1. W. Zhang, X. Zhou, and Y. Tan, "Analysis of the range false target deception jamming effect against fire control radar," Sichuan Ordnance Journal, 28(4), (2007).

2. S. C. Luo, "An algorithm of deception jamming suppression based on blind signal separation," Journal of Electronics \& Information Technology, 33(12), 2801-2806, (2011).

3. G. Lu, B. Tang, and G. Gui, "Deception ECM signals cancellation processor with joint timefrequency pulse diversity," IEICE Electronics Express, 8(19), 1608-1613, (2011).

4. R. Chen and Y. M. Wang, "Detection of frequencyshifting jamming to LFM pulse-compression radar based on FRFT," Radar Science \& Technology, (2013).

5. S. Peleg and B. Porat, "Linear FM signal parameter estimation from discrete-time observations," IEEE Trans on Aerospace and Electronic Systems, 27(4), 607-616, (1991).

6. R. T. Behrens and L. L. Scharf, "Signal processing applications of oblique projection operators," IEEE Transactions on Signal Processing, 42(6), 1413-1424, (1994).

7. R.T. Behrens and L. L. Scharf, " Corrections to 'signal processing applications of oblique projection operators' [Correspondence],” IEEE Trans. on Signal Processing, 44(5), 1300, (1996).

8. X. J. Yang, "Theory and applications of local fractional fourier analysis," Chinese Journal of Mechanical Engineering, 1(4), (2012).

9. D. Curtis Schleher. Electronic warfare in the information age, MA: Artech House, Norwood,Massachusetts, (1999).

10. M. L. Mccloud and L. L. Scharf, "A new subspace identification algorithm for high-resolution DOA estimation," IEEE Transactions on Antennas \& Propagation, 50(10), 1382-1390, (2002). 